\title{
CORRELATIONS OF SELF-BOUND IDENTICAL FERMIONS
}

\author{
G.P. Kamuntavičius ${ }^{\text {a }}$, A. Mašalaite ${ }^{a}$, S. Mickevičius ${ }^{\text {a }}$, D. Germanas ${ }^{\text {b }}$, \\ R.-K. Kalinauskas ${ }^{\mathrm{b}}$, and R. Žemaičiūniené ${ }^{\mathrm{c}}$ \\ a Vytautas Magnus University, Donelaičio 58, LT-44248 Kaunas, Lithuania \\ E-mail: g.kamuntavicius@gmf.vdu.lt \\ ${ }^{\mathrm{b}}$ Institute of Physics, Savanoriu 231, LT-02300 Vilnius, Lithuania \\ c Šiauliai University, Vilniaus 88, LT-76285 Šiauliai, Lithuania
}

Received 15 September 2006

\begin{abstract}
A method for the calculation of translationally invariant wave functions for systems of identical fermions with arbitrary potential of pair interaction is developed. It is based on the well-known result that the essential dynamic part of Hamiltonian for the system of identical particles is the reduced Hamiltonian operator describing a relative movement of two particles inside the system. The eigenfunctions of this operator take into account all correlations caused by interaction. These eigenfunctions are basic elements for building the components (i. e., the functions with a lower degree of antisymmetry) used to construct the total antisymmetric wave function of the system. The main problem of this approach appears to be the antisymmetrization of the components. The developed universal algorithm for antisymmetrization gives a possibility to carry out this operation in a simple way and to keep numerical approximations under control.
\end{abstract}

Keywords: self-bound systems, identical fermions, translational invariance, antisymmetrized states, reduced Hamiltonian

PACS: 03.65.Ca, 03.65.Fd, 21.60.-n

\section{Introduction}

The wave function of a self-bound system in absence of external fields must be invariant in respect of spatial translations. This invariance of wave function means that it is dependent only on intrinsic degrees of freedom of the system. However, the traditional methods for description of quantum systems, such as the shell model or the Hartree-Fock self-consistent field (SCF) method, produce wave functions dependent on a set of one-particle variables, hence also on the system centreof-mass radius-vector. This shortcoming of the mentioned methods is well known, however, the wave functions dependent on one-particle variables are very attractive because they allow a simple procedure of antisymmetrization. In some cases this approximation with the Hamiltonian and the wave functions, both dependent on redundant variables, do not pose any serious problems. The uncontrollably moving and excited centre of mass of a system such as an atom, a molecule, or an electron gas in a solid is not a problem for the system under consideration (i. e., the system of electrons) but the problem of external fields, which keep these electrons together to form such a system. However, for nuclei and hadrons the translational invariance appears to be a real problem. These systems are essentially selfbound. The external fields, normally able to dictate behaviour of such a system, are very weak in comparison with interactions of nucleons or quarks, i. e., the structural elements of nuclei or hadrons. The translational invariance of corresponding wave functions creates less investigated additional correlations between particles.

A quantum system without correlations is a system whose wave function can be presented as a product of wave functions of subsystems composing the entire system. Such factorization of wave function is possible when interactions among these subsystems are absent or can be described by some central field forcing these subsystems to compose the system. Such systems do not exist, this is only a more or less acceptable model simplifying the description. If particles compose a system, they always are correlated, and first of all due to interactions among them. However, besides dynamic correlations, there exist additional correlations, characteristic especially of quantum systems. These are correlations created by different symmetries of the system. Any symmetry has a corresponding operator commuting with a Hamiltonian. Wave function of the quantum system must be an eigenfunction of these operators. This introduces new quantum numbers charac- 
terizing the system, but at the same time requires linear combinations of mentioned products of subsystem functions, hence additional correlations.

The first among them is invariance of the system in respect of rotations and inversion. The corresponding quantum numbers are angular momentum and isospin, projections of these quantum numbers, and parity. The second invariance is invariance of the system in respect of identical particle permutations. For the system composed of identical fermions, this symmetry is known as the Pauli principle. The latter kind of correlations is created by the mentioned above translational invariance. At any kind of correlations taken into account, one gains some quantum numbers for the entire system, but at the same time the particles lose individuality and none of them can be described by a complete set of necessary quantum numbers. For example, by coupling the momenta one loses momentum projections for individual particles. Antisymmetrizing the function, the determinants appear, states for individual particles are not defined at all. Only the configuration, i.e., a set of restricted quantum numbers characterizing the entire system, is defined. For a translationally invariant function one cannot define even the configuration.

Some methods recently developed for few-body systems with strong interaction are designed to produce translationally invariant wave functions. These are the methods based on the Faddeev or Faddeev-Jakubovsky equations [1], on the Green's function Monte Carlo (GFMC) method [2], or on the expansions in a large basis of harmonic oscillator functions [3]. In the first case, the translational invariance of a wave function arises from equations written in intrinsic variables. For GFMC the translational invariance is given by infrastructure of variational wave function, spatial part of which is composed of Jastrow multipliers dependent on translationally invariant differences of one-particle coordinates. The last mentioned calculations operate with the harmonic oscillator wave functions as a basis for realistic calculations. These functions, if a complete basis of ones corresponding to a given number of oscillator quanta is taken into account, can be projected to superpositions with an unexcited centre of mass of the system.

The goal of the current study is a new efficient method of construction of the translationally invariant wave functions for the system of identical fermions with arbitrary potential of pair interaction. These functions are eigenfunctions of an intrinsic Hamiltonian, i. e., the Hamiltonian dependent on intrinsic variables. One ensures translational invariance for wave functions by taking as arguments the set of translationally invariant spatial variables, so-called Jacobian variables, defined as in [4]. The formalism is free of any phenomenological parameters (like variational parameters in GFMC) or fields (like in any model exploiting the shell model idea), as well as of any "effective interactions".

The basic point of the method is the well-known result that the essential dynamic part of Hamiltonian for the system of identical particles is the reduced Hamiltonian $(\mathrm{RH})$ operator $[5,6]$. The system of eigenfunctions of this operator takes into account all correlations caused by interaction. The Schrödinger equation for RH operator is very simple, not more complex than the Schrödinger equation for relative movement of two particles interacting by a given potential. This really looks like a one-particle problem, like in the shell model. These eigenfunctions are basic in our formalism to the wave function construction. The main problem in this approach appears to be the antisymmetrization of a component (i.e., the construct with a lower degree of antisymmetry) of a wave function. The developed universal algorithm for antisymmetrization gives us a possibility to perform this operation in a simple way and to keep numerical approximations under control. In the mentioned basis with correlations taken into account in advance the convergence is fast.

In Sec. 2 we present the basic definitions and introduce the problem under consideration. Section 3 is devoted to description of the new original procedure for antisymmetrization of wave functions for translationally invariant systems composed of identical fermions. In Sec. 4 one considers the entire problem based on factorization of the Hamiltonian matrix. The summary of the developed method, numerical test, and conclusions are given in Sec. 5.

\section{Correlated components of wave function}

The suggested consideration is based on the presentation of an intrinsic Hamiltonian for the system of $N$ identical particles as a sum of $N(N-1) / 2$ two-particle operators:

$$
\begin{gathered}
\mathcal{H}=-\frac{\hbar^{2}}{2 m} \sum_{i=1}^{N} \nabla_{i}^{2}+\frac{\hbar^{2}}{2 m N} \nabla_{0}^{2} \\
+\sum_{i, j=1(i<j)}^{N} V\left(\mathbf{r}_{i}-\mathbf{r}_{j}, \sigma_{i} \sigma_{j}\right)
\end{gathered}
$$




$$
\begin{aligned}
& =\sum_{i, j=1(i<j)}^{N}\left[-\frac{\hbar^{2}}{2 m N}\left(\nabla_{i}-\nabla_{j}\right)^{2}+V\left(\mathbf{r}_{i}-\mathbf{r}_{j}, \sigma_{i} \sigma_{j}\right)\right] \\
& \equiv \sum_{i, j=1(i<j)}^{N} h_{i, j}
\end{aligned}
$$

where $V\left(\mathbf{r}_{i}-\mathbf{r}_{j}, \sigma_{i} \sigma_{j}\right)$ is the potential of interaction, dependent, as usual, on difference of radius-vectors of interacting particles $i$ and $j$ and on discrete degrees of freedom $\sigma_{i}, \sigma_{j}$ of these particles, such as spins, isospins, etc. The term $\hbar^{2} \nabla_{0}^{2} / 2 m N$ is the kinetic energy operator of the centre of mass of the system.

The arbitrary eigenvalue of this Hamiltonian, due to antisymmetry of corresponding eigenfunction, equals the number of terms present in the sum multiplied by the expectation value of one of these operators, i. e.,

$$
\begin{aligned}
& \mathcal{E}=\langle\mathcal{H}\rangle=\left(\begin{array}{c}
N \\
2
\end{array}\right)\left\langle-\frac{\hbar^{2}}{m N} \nabla_{\xi_{i, j}}^{2}+V\left(\sqrt{2} \xi_{i, j}, \sigma_{i} \sigma_{j}\right)\right\rangle \\
& \equiv\left(\begin{array}{c}
N \\
2
\end{array}\right)\left\langle h_{i, j}\right\rangle \equiv\left\langle H_{i, j}\right\rangle .
\end{aligned}
$$

Here $\left(\begin{array}{c}N \\ 2\end{array}\right)=N(N-1) / 2$ is a binomial coefficient and $\xi_{i, j}=\left(\mathbf{r}_{i}-\mathbf{r}_{j}\right) / \sqrt{2}$ is one of the Jacobian variables. To simplify the expressions, let us take defined values for indices, namely $i=N-1$ and $j=N$, and mark the corresponding Jacobian variable as $\xi \equiv \xi_{N-1, N}$. The operator $H_{N-1, N} \equiv H$ is called the reduced Hamiltonian operator and is the main in further consideration. The best way to eliminate dimensions of physical variables in above expressions is to present the multiplier $\hbar^{2} / m$ in the form

$$
\frac{\hbar^{2}}{m}=\hbar \omega \cdot b^{2}
$$

where $\hbar \omega$ can be considered as a parameter of energy and $b$ as a length parameter. Really, one free parameter appears here, $\omega$ or $b$. Let us consider below the dimensionless values for energy $\mathcal{E} \rightarrow \mathcal{E} / \hbar \omega$ and variable $\xi \rightarrow \xi / b$. The value 1 may certainly be assigned for parameter $b$. Finally, the dimensionless reduced Hamiltonian is

$$
H=\left(\begin{array}{c}
N \\
2
\end{array}\right)\left[-\frac{1}{N} \Delta_{\xi}+\frac{1}{\hbar \omega} V\left(\sqrt{2} \xi, \sigma_{N-1} \sigma_{N}\right)\right] .
$$

Thus, the expression for the eigenvalue of Hamiltonian in terms of $\hbar \omega$ is $\mathcal{E}=\langle H\rangle$.

This is a very interesting result stating that calculation of eigenvalue does not require the total Hamilton operator. Only the significant part of the many-particle Hamiltonian with pair interaction - the two-particle RH operator - is necessary. However, practical application of simplifications caused by this observation is not easy. Due to noncommutation of $H$ and $\mathcal{H}$ operators and antisymmetry of a wave function one cannot prescribe any set of quantum numbers for the state of the last two particles. The way of solving this problem was found years ago, when coefficients of fractional parentage (CFP) for the atomic shell model were introduced $[7,8]$. These are the coefficients of the antisymmetric wave function expansion in terms of linear combinations of some less complex structures with a lower degree of antisymmetry, later on called the components of a wave function. For the problem under consideration the components are products of the antisymmetric function for the last two particles, dependent on variables present in the given reduced Hamiltonian operator, and the antisymmetric function dependent on a set of all the remaining variables [6]. In other words, the idea of coefficients of fractional parentage realizes the antisymmetrization in space of quantum numbers of the complete system of components. Let us mark the antisymmetric wave function for the $N$ particle system as

$$
\Psi_{\mathcal{E} \Lambda M}(1,2, \ldots, N-1, N),
$$

where $\mathcal{E}$ is the corresponding eigenvalue of Hamiltonian, $\Lambda$ is a set of "good" quantum numbers, such as momentum, parity, isospin, etc., and $M$ is a set of corresponding projections of momenta quantum numbers.

The mentioned construct with a lower degree of antisymmetry, i. e., the component of wave function, is

$$
\begin{aligned}
& \Phi_{(\bar{\Gamma} \bar{\Lambda}, \varepsilon \rho) \Lambda M}(1,2, \ldots ; N-1, N) \\
& =\sum_{\bar{M} \mu} \Psi_{\bar{\Gamma} \bar{\Lambda} \bar{M}}(1,2, \ldots, N-2) \\
& \times \varphi_{\varepsilon \rho \mu}(N-1, N)\left[\begin{array}{ccc}
\bar{\Lambda} & \rho & \Lambda \\
\bar{M} & \mu & M
\end{array}\right] .
\end{aligned}
$$

It is a coupled momenta function with exact quantum numbers $\Lambda M$ coinciding with ones for complete wave function. The function $\Psi$ present on the right-hand side of equation is a "spectator" function dependent on variables of first $N-2$ particles. $\bar{\Lambda} \bar{M}$ is a set of corresponding quantum numbers and their projections. $\bar{\Gamma}$ marks a set of all the remaining quantum numbers necessary to ensure completeness and orthonormality of basis of "spectator" functions. The second function $\varphi$ is an eigenfunction of the RH operator, hence it is best to choose the quantum numbers $\rho$ and $\mu$ as a complete set 
of eigenvalues of operators commuting with the RH operator. $\varepsilon$ marks an eigenvalue of the Schrödinger equation for RH. The last factor is the product of ClebschGordan coefficients for momenta and Kronecker deltas for parities, isospin projections, etc.

The most interesting for us are the coefficients of the wave function expression in terms of a complete set of components:

$$
\begin{aligned}
& \Psi_{\mathcal{E} \Lambda M}(1,2, \ldots, N-1, N) \\
& =\sum_{\bar{\Gamma} \bar{\Lambda}, \varepsilon \rho}\langle\mathcal{E} \Lambda \| \bar{\Gamma} \bar{\Lambda}, \varepsilon \rho\rangle \\
& \times \Phi_{(\bar{\Gamma} \bar{\Lambda}, \varepsilon \rho) \Lambda M}(1,2, \ldots ; N-1, N) .
\end{aligned}
$$

The system of equations for these coefficients consists of the Schrödinger equation and the condition that a wave function of the system composed of identical fermions must be an eigenfunction of the antisymmetrization operator corresponding to the eigenvalue equal to one:

$$
\begin{gathered}
(\mathcal{H}-\mathcal{E}) \Psi_{\mathcal{E} \Lambda M}(1,2, \ldots, N-1, N)=0, \\
(A-1) \Psi_{\mathcal{E} \Lambda M}(1,2, \ldots, N-1, N)=0 .
\end{gathered}
$$

Obviously, the antisymmetrizer present in Eq. (9) must be properly normalized, i. e.

$$
A=\frac{1}{N !} \sum_{P \in S_{N}} \delta_{P} P,
$$

where $P$ are permutation operators of symmetric group $S_{N}$ and $\delta_{P}$ is the parity of permutation $P$. It is due to this normalization that the antisymmetrizer is a projection operator, i. e., satisfies the condition $A A=A$. The same condition for matrix of this operator simplifies further consideration.

However, simultaneous solution of both equations is very problematic. As usual in quantum mechanics, first of all one needs to construct a complete set of solutions for a simpler equation and later on, applying these functions, to construct solutions of a more complex equation. The simpler equation in our case is the second equation, i. e. Eq. (9), so first of all it is necessary to antisymmetrize the components. The antisymmetrized basic functions, eigenfunctions of antisymmetrizer, are

$$
\begin{aligned}
& \Psi_{\Gamma \Lambda M}(1,2, \ldots, N-1, N)=\sum_{\bar{\Gamma} \bar{\Lambda}, \varepsilon \rho}\langle\Gamma \Lambda \| \bar{\Gamma} \bar{\Lambda}, \varepsilon \rho\rangle \\
& \times \Phi_{(\bar{\Gamma} \bar{\Lambda}, \varepsilon \rho) \Lambda M}(1,2, \ldots ; N-1, N) .
\end{aligned}
$$

Specifically, the coefficients present in this expansion are the coefficients of fractional parentage; $\Gamma$ is an additional quantum number necessary to mark the antisymmetric basic functions. The last operation is a diagonalization of the Hamiltonian operator while applying this basis of functions. For this, one needs to define the expansion of wave function

$$
\begin{aligned}
& \Psi_{\mathcal{E} \Lambda M}(1,2, \ldots, N-1, N) \\
& =\sum_{\Gamma}\langle\mathcal{E} \mid \Gamma\rangle \Psi_{\Gamma \Lambda M}(1,2, \ldots, N-1, N)
\end{aligned}
$$

and to solve an algebraic eigenvalue equation for these coefficients:

$$
\sum_{\Gamma^{\prime}}\left[\left\langle\Gamma|\mathcal{H}| \Gamma^{\prime}\right\rangle-\mathcal{E}\right]\left\langle\mathcal{E} \mid \Gamma^{\prime}\right\rangle=0 .
$$

The final expression for wave function in terms of components with a lower degree of antisymmetry looks like

$$
\begin{aligned}
& \Psi_{\mathcal{E} \Lambda M}(1,2, \ldots, N-1, N) \\
& =\sum_{\Gamma}\langle\mathcal{E} \mid \Gamma\rangle \sum_{\bar{\Gamma} \bar{\Lambda}, \varepsilon \rho}\langle\Gamma \Lambda \| \bar{\Gamma} \bar{\Lambda}, \varepsilon \rho\rangle \\
& \times \Phi_{(\bar{\Gamma} \bar{\Lambda}, \varepsilon \rho) \Lambda M}(1,2, \ldots ; N-1, N) .
\end{aligned}
$$

\section{Antisymmetrization in correlated basis}

Let us first of all formulate the well-known results concerning antisymmetrization of different wave functions in a way giving possibility to generalize it for translationally invariant functions.

The best known procedure is antisymmetrization of many-particle function dependent on one-particle spatial variables and intrinsic degrees of freedom, when particles do not interact, correlations are absent, and wave functions split into product of one-particle functions. In this case, antisymmetrization can be performed in given configuration, i. e., for each set of oneparticle states, provided that coinciding one-particle states are absent. This construct equals the determinant composed of mentioned one-particle functions with permuted one-particle variables.

The simplest fractional parentage expansion for this wave function is expansion for determinant in minors, i. e., in the constructs antisymmetric only in respect of permutations of the first $N-1$ particles. The last particle is not antisymmetrized with particles composing this "spectator" system. However, a state for this particle in an antisymmetrized function runs over all 
the states present in configuration under investigation. The coefficients of expansion for antisymmetric function, equal to the determinant of the $N$ th order in terms of minors of $N-1$ order, are the one-particle CFP. They are well-known from linear algebra. Due to normalization of wave function and all minors they equal $\pm 1 / \sqrt{N}$. In this case, definition for the two-particle CFP is completely analogous. They are coefficients present in the same determinant expansion in terms of minors of the $N-2$ order multiplied by antisymmetric wave function for remaining two particles.

The antisymmetrization and fractional parentage expansion for more complex function with coupled angular momentum-like (orbital, spin, isospin, etc.) quantum numbers can be formulated in analogous way. In this case, there appear shells, i.e., groups of particles having the same one-particle radial wave function but different projections of orbital and intrinsic momenta of particles. However, the antisymmetrization for such a function as in previous case is impossible. The operation of momenta coupling results in sums over projections of quantum numbers, hence a complete set of one-particle variables for every particle stays undefined and the old scheme of antisymmetrization cannot work. Specifically, the antisymmetrization in the defined shell creates traditional coefficients of fractional parentage. As in the previous case, they are independent of one-particle radial wave functions, hence are universal. For more complex systems, containing more than one shell, antisymmetrization is not so complicated as believed because radial functions for different shells are orthogonal, and this significantly simplifies consideration. The CFP, defined for such a complex configuration, are expressible in terms of traditional CFP and transformation matrices. Again, in this case antisymmetrization can be performed for defined configuration, it is in finite space of components basis.

Antisymmetrization for translationally invariant wave function is a very complex problem in comparison with considered ones. In the form given below, it is introduced in [6] and still applied only to threedimensional harmonic oscillator functions [3]. This is the simplest application of the proposed scheme because only for harmonic oscillator basis this operation can be performed in finite space of basic component functions. The reason for this is a very simple structure of the many-particle harmonic oscillator Hamiltonian, invariant in respect of any permutations of Jacobian variables. This symmetry creates a conserving quantum number - a total number of oscillator quanta proportional to the eigenvalue of such a Hamiltonian, and the possibility to perform antisymmetrization separately for each value of this integer number. However, this simplification is not so useful as it looks at a first glance because in many cases long expansions in this basis are necessary and convergence of expansions is very slow.

The procedure one applies is defined in a universal way and is based on calculation of the antisymmetrizer matrix in components basis and spectral decomposition of this matrix in the case when the eigenfunctions of the RH operator are applied as "actor" functions. The formalism is devoted to description of bound states of the quantum system, so in the expansion of a component one can apply square-integrable functions for a "spectator", as well as square-integrable functions for an "actor". Obviously, in most interesting applications RH has both discrete and continuous eigenvalues. The necessary basis for the corresponding Sturm-Liouville problem can be constructed taking proper boundary conditions for eigenfunctions, for example, taking all eigenfunctions equal to zero at some value of argument larger than a characteristic measure for the system dimensions.

The next problem is a complete system of functions for "spectator". For these functions one cannot formulate any equations, hence one is free in choice. The simplest translationally invariant basis, as mentioned above, is basis of three-dimensional harmonic oscillator functions, enriched by intrinsic degrees of freedom of particles.

Once the construction of basis for components is completed, one can start calculating the matrix of an antisymmetrization operator. If, as defined, one has antisymmetrized "spectator" as well as "actor" functions and is going to apply this operator to the matrix calculation, the best factorization for the antisymmetrization operator is as follows [9]:

$$
\begin{aligned}
A_{1,2, \ldots, N}= & A_{N-1, N} A_{1,2, \ldots, N-2} Y_{1,2, \ldots, N-2 ; N-1, N} \\
& \times A_{1,2, \ldots, N-2} A_{N-1, N}
\end{aligned}
$$

where

$$
\begin{aligned}
& Y_{1,2, \ldots, N-2 ; N-1, N}= \\
& \left(\begin{array}{c}
N \\
2
\end{array}\right)^{-1}\left[1-(N-2) P_{N-2, N}\right. \\
& \left.+\left(\begin{array}{c}
N-2 \\
2
\end{array}\right) P_{N-3, N-1} P_{N-2, N}\right]
\end{aligned}
$$

and $P_{i, j}$ are transposition operators. 
The normalization of antisymmetrizer given in Eq. (10) makes the corresponding matrix idempotent or projection matrix:

$$
\mathbf{A A}=\mathbf{A} .
$$

This matrix is real and symmetric $\left(\mathbf{A}^{+}=\mathbf{A}\right)$. Obviously, its eigenvalues are equal to ones and zeroes. Hence, the sum of eigenvalues of this matrix equals the number of eigenvalues equal to one. By definition, it coincides with a trace of matrix $\mathbf{A}$ and defines the rank $r$ of this matrix. Eigenvectors corresponding to zero eigenvalues do not have a physical meaning. Antisymmetric states can be described only by eigenvectors corresponding to the eigenvalue equal to one. This matrix has an attractive feature - each entry $a_{i j}$ of this matrix equals the product of the $i$ th and $j$ th rows (columns) of this matrix. Hence, each diagonal entry of matrix equals the sum of squares of entries of this row or column. This is a useful measure of projectiveness of any calculated part of matrix. Moreover, if some diagonal entry of this matrix equals zero, all entries of corresponding row and column are identical zeroes. This means that such a basic component must be removed from basis because it has some additional symmetries and cannot be antisymmetrized. The second conclusion from this feature of the projection matrix is that if some diagonal entry of this matrix equals one, again all the remaining entries of corresponding row and column are equal to zero. This means that the corresponding basic component is already antisymmetric and needs no additional antisymmetrization.

The spectral decomposition for this matrix is

$$
\mathbf{A}=\mathbf{F F}^{+},
$$

where $\mathbf{F}$ is the matrix composed of eigenvectors, corresponding to unit eigenvalues, as columns. Every column of this matrix defines antisymmetric wave function for $N$ particles. Matrix $\mathbf{F}$ has $n$ rows ( $n$ equals the order of matrix $\mathbf{A}$ ) and $r$ columns. Every column is normalized and they are orthogonal, i. e.,

$$
\mathbf{F}^{+} \mathbf{F}=\mathbf{1} \text {. }
$$

The entries of matrix $\mathbf{F}$ are precisely the coefficients of fractional parentage for any of the mentioned components bases. For this matrix construction, the observation that every column of matrix $\mathbf{A}$ is an eigenvector of this matrix, corresponding to the eigenvalue equal to one, is very useful. However, the number of these eigenvectors exceeds the rank, hence some of them are linearly dependent. Moreover, they are not orthonormal vectors, but direct calculation of matrix $\mathbf{F}$ is not the problem due to the well-known result that each symmetric matrix of an order $n$ and a rank $r$ can be written in a form

$$
\mathbf{A}=\left(\begin{array}{cc}
\mathbf{Z} & \mathbf{Q}^{+} \\
\mathbf{Q} & \mathbf{Q Z}^{-1} \mathbf{Q}^{+}
\end{array}\right),
$$

where $\mathbf{Z}$ is a non-degenerate submatrix of the order $r$. The matrix of spectral decomposition for matrix $\mathbf{A}$ is

$$
\mathbf{F}=\left(\begin{array}{c}
\mathbf{Z}^{1 / 2} \\
\mathbf{Q} \mathbf{Z}^{-1 / 2}
\end{array}\right) .
$$

The normalization condition for $\mathbf{F}$ looks like

$$
\mathbf{F}^{+} \mathbf{F}=\mathbf{Z}^{+} \mathbf{Z}^{-1 / 2} \mathbf{Q}^{+} \mathbf{Q Z}^{-1 / 2}=\mathbf{1} .
$$

It can be written in the form

$$
\mathbf{Q}^{+} \mathbf{Q}=\mathbf{Z}(\mathbf{1}-\mathbf{Z}) \text {. }
$$

\section{Eigenvalues of Hamiltonian matrix}

Once the matrix $\mathbf{F}$ is constructed, one is ready to obtain a matrix of the total Hamiltonian. As it was mentioned, first of all one must take into account that this matrix due to identity of particles equals the matrix of the RH operator. Having in mind that the coefficients of the antisymmetric function expression in terms of components are CFP, i. e., entries of $\mathbf{F}$ matrix, one can present the matrix of the Hamiltonian in a form

$$
\mathcal{H}=\mathbf{F}^{+} \mathbf{H F} .
$$

Here the matrix of the RH operator is given in components basis. This is our goal because in this basis matrix $\mathbf{H}$ is diagonal. The best choice is to rearrange the entries of this matrix in the nonvanishing order. By the way, this ordering of basic functions looks very natural and means that one takes the function with "actor" being in the lowest possible state as the first basic function, then the one in the first excited state as the second basic function, etc. Obviously, for every "actor" function one has to take a necessary set of "spectator" functions.

The order of the Hamiltonian matrix equals rank $r$ of matrix of an antisymmetrizer, but the order of the diagonal matrix of the RH operator is larger, equal to the order of the antisymmetrizer matrix $n$ or, in other words, the number of basic components taken into account. This infrastructure of the Hamiltonian matrix is very useful in cases when dimensions of matrices present in expression are very large. Taking into account this infrastructure, one can construct different approximations for eigenvalues and eigenfunctions. The 
idea is as follows. The antisymmetric states involved in the expansion are given by columns of matrix $\mathbf{F}$. Let the rank of this matrix be equal to one, this means it contains only one column. In this case the eigenvalue of the Hamiltonian equals

$$
\begin{aligned}
\mathcal{E} & =\varepsilon_{1} f_{11}^{2}+\varepsilon_{2} f_{21}^{2}+\ldots+\varepsilon_{n} f_{n 1}^{2} \\
& \equiv \varepsilon_{1} \omega_{1}+\varepsilon_{2} \omega_{2}+\ldots+\varepsilon_{n} \omega_{n} .
\end{aligned}
$$

Here $\varepsilon_{i}$ are the eigenvalues of the $\mathrm{RH}$ operator arranged in the nonvanishing order. Due to reality of matrix $\mathbf{F}$ the squares of entries are the probabilities (due to normalization this sum of squares equals one, i.e., $\sum_{i=1}^{n} f_{i 1}^{2}=1$ ) of different states of the $\mathrm{RH}$ operator in the expansion for the energy eigenvalue of the total Hamiltonian. The expansion of the same kind can be obtained for each eigenvalue after matrix diagonalization because the corresponding eigenvector equals a linear combination of columns of matrix $\mathbf{F}$ :

$$
\mathcal{E}=\sum_{i=1}^{n} \varepsilon_{i} \omega_{i}, \quad \sum_{i=1}^{n} \omega_{i}=1, \quad \omega_{i} \geq 0 .
$$

As it follows from definitions, all $\omega_{i}$ are nonnegative. At the same time some first eigenvalues of $\mathrm{RH}$ are negative and the remaining ones are positive. To minimize the value for energy one needs the values of probabilities corresponding to negative values of epsilons to be as maximal as possible. Exactly the possibility to obtain this formula for the eigenvalue of the manyparticle Hamiltonian is basic to the Hall-Post lower bounds for energies construction [5]. This lower bound can be obtained taking a maximal value of probability corresponding to the lowest value of RH energy. This means that

$$
E_{\text {Hall-Post }}=\varepsilon_{1} .
$$

Obviously, this is exactly a lower bound because

$$
\begin{aligned}
E-E_{\text {Hall-Post }} & =\sum_{i=1}^{n} \varepsilon_{i} \omega_{i}-\varepsilon_{1} \sum_{i=1}^{n} \omega_{i} \\
& =\sum_{i=2}^{n}\left(\varepsilon_{i}-\varepsilon_{1}\right) \omega_{i}>0 .
\end{aligned}
$$

On the other hand, our consideration has shown that this lower bound value for energy corresponds to wave function equal to the first basic component. This component is not antisymmetric, hence the corresponding eigenvalue is significantly lower than that for the antisymmetrized function.

The real problem in our task is a huge amount of basic states while describing strongly correlated systems and as consequence - a very big rank of matrix of the antisymmetrizer, hence a large order of the Hamiltonian matrix. One needs the best possible values for energy and the corresponding high quality wave functions at the lowest possible price. The recommendations for successful realization of this program are as follows.

First of all, one does not need the complete calculation of matrix of the antisymmetrizer A. As one may conclude from this matrix presentation, Eq. (20), for complete matrix and its spectral decomposition construction only $r$ linearly independent columns or rows of this matrix, i.e., matrices $\mathbf{Z}$ and $\mathbf{Q}$, are necessary. These define the matrix of spectral decomposition $\mathbf{F}$, Eq. (21), necessary for further calculations. During calculations of this part of matrix $\mathbf{A}$ one can control how far this calculation is from finish because due to the mentioned above condition the sum of squares of entries of column must equal the corresponding diagonal entry. When this condition is fulfilled with necessary precision, one can stop calculations for this column and start calculating the next column. The corresponding submatrix $\mathbf{Z}$ will show how many columns one needs. As it is required, this matrix must be non-degenerate, hence its determinant must be nonzero. Only in this case, one can continue column calculations. If the determinant equals zero, the basic state, corresponding to column under consideration, is linearly dependent on already calculated columns. This statement follows from relation for entries of projection matrix, stating that each submatrix of the projection matrix equals the Sturm matrix of corresponding rows or columns. Hence, the determinant of submatrix $\mathbf{Z}$ is the best indicator of the linear dependence of rows (columns) of projection matrix. Finally, one can check the rank of matrix. It must equal the trace.

The last operation, after both matrices, $\mathbf{F}$ and $\mathbf{H}$, are calculated, is the construction of the Hamiltonian matrix and its diagonalization. This is the standard operation. However, in many applications only a few lowest eigenvalues and eigenvectors of this matrix are necessary. The method of obtaining converging approximations to exact values for a few lower states avoiding calculation of the complete Hamiltonian matrix is developed. The idea is based on applied transparent presentation for the Hamiltonian. As usual, the diagonalization of this matrix requires the set of orthogonal transformations

$$
\mathbf{R}^{+} \mathbf{F}^{+} \mathbf{H F R}=(\mathbf{F R})^{+} \mathbf{H F R} .
$$

In our approach the transformation for the entire matrix is not necessary - one can transform the matrix 
$\mathbf{F}$ as needed. The necessary transformations are products of $r-1$ Householder reflections [10], i.e., $\mathbf{R}=\mathbf{R}_{1} \mathbf{R}_{2} \ldots \mathbf{R}_{r-1}$, transforming the first, the second, and all the following rows of matrix $\mathbf{F}$ to convert it into the lower triangle form. To some extent this way of matrix transforming looks like the well-known QR decomposition of a matrix, see [10], into an orthogonal matrix $\mathbf{Q}$ and upper triangular matrix $\mathbf{R}$. If the first row of matrix $\mathbf{F}$ is $\mathbf{f}_{1}^{+}=\left(f_{11} f_{12} \ldots f_{1 r}\right)$, the first Householder matrix, whose order equals $r$, is

$$
\mathbf{R}_{1}=\mathbf{1}-2 \frac{\mathbf{f}_{1} \mathbf{f}_{1}^{+}}{\mathbf{f}_{1}^{+} \mathbf{f}_{1}} .
$$

The second Householder matrix is

$$
\mathbf{R}_{2}=\left(\begin{array}{cc}
\mathbf{1}_{1} & \mathbf{0}^{+} \\
\mathbf{0} & \mathbf{1}-2 \frac{\mathbf{f}_{2} \mathbf{f}_{2}^{+}}{\mathbf{f}_{2}^{+} \mathbf{f}_{2}}
\end{array}\right),
$$

where $\mathbf{f}_{2}^{+}=\left(f_{22} f_{23} \ldots f_{2 r}\right)$ is the upper (right) part of the second row of matrix $\mathbf{F}$ and the order of unit submatrix is shown. The last matrix of transformation is

$$
\mathbf{R}_{r-1}=\left(\begin{array}{cc}
\mathbf{1}_{r-2} & \mathbf{0}^{+} \\
\mathbf{0} & \mathbf{1}-2 \frac{\mathbf{f}_{r-1} \mathbf{f}_{r-1}^{+}}{\mathbf{f}_{r-1}^{+} \mathbf{f}_{r-1}}
\end{array}\right) .
$$

Here $\mathbf{f}_{r-1}^{+}=\left(f_{r-1 r} f_{r r}\right)$. After these transformations are performed, the new Hamiltonian matrix $\mathbf{F}^{\prime+} \mathbf{H F}^{\prime}$ appears, where $\mathbf{F}^{\prime}=\mathbf{F R}_{1} \mathbf{R}_{2} \ldots \mathbf{R}_{r-1}$. Obviously, the new matrix has the same eigenvalues as the initial one. Moreover, $\mathbf{F}^{\prime+} \mathbf{F}^{\prime}=\mathbf{1}$ and $\mathbf{F}^{\prime} \mathbf{F}^{\prime+}=\mathbf{A}$. The matrix $\mathbf{F}^{\prime}$ has zeros in the upper triangle and maximal possible diagonal entries, equal to norms of parts of entries of rows, involved in the Householder reflection, i.e., $\mathbf{f}_{1}^{+} \mathbf{f}_{1}, \mathbf{f}_{2}^{+} \mathbf{f}_{2}, \ldots, \mathbf{f}_{r-1}^{+} \mathbf{f}_{r-1}$, respectively.

Now one can obtain that only the first column of matrix $\mathbf{F}^{\prime}$ has a nonzero entry in the first row. This entry squared equals the maximal weight (probability) of the lowest eigenvalue of RH. Any combinations of this column with remaining columns of matrix $\mathbf{F}^{\prime}$ can only lower this probability. The nonzero weight (probability) of the first and second of the lowest eigenvalues of RH can produce only the first and second columns, etc. The set of negative eigenvalues of RH, as mentioned, is finite. Let this number be equal to $k$. Not more than $k-1$ Householder reflections are necessary to obtain an acceptable result because, as already mentioned, each column with zero entries, corresponding to negative $\mathrm{RH}$ eigenvalues, while combining with the first $k$ columns, can only lower the probabilities of negative epsilons, hence a negative part of the eigenvalue for the total Hamiltonian.

\section{Summary and conclusions}

Summarizing, let us present the list of consequent steps for the application of the proposed method.

The first step is construction of the reduced Hamiltonian operator given in Eq. (4) and generation of the complete system of square-integrable functions for this operator. The spectrum of this Sturm-Liouville problem can be discretized by applying simple boundary conditions for eigenfunctions.

The next step is construction of the system of basic functions for components, taking arbitrary antisymmetric functions for "spectator" and eigenfunctions of RH for "actor" basis. The components must be arranged in the order corresponding to nonvanishing diagonal entries of matrix $\mathbf{H}$, i. e., eigenvalues of the RH operator.

Now one can start calculation of the antisymmetrizer matrix using factorization given in Eq. (15). This representation is optimal for component basis because due to antisymmetry of "spectator" and "actor" functions both antisymmetrizers present on the left-hand side as well as both antisymmetrizers present on the right-hand side may be omitted. Obviously, one must calculate only matrices of two nontrivial operators: $P_{N-2, N}$ and $P_{N-3, N-1} P_{N-2, N}$. Later on, combining these with the identity matrix according to Eq. (16), one can construct the matrix of the antisymmetrizer. Due to simple structure of this matrix, thoroughly considered in Sec. 3, one needs only a part of this matrix, necessary for matrix $\mathbf{F}$ construction according to formulas given in Eqs. (20) and (21). By the way, one can start calculations of eigenvalues for the total Hamiltonian at any number of columns (rows) of the antisymmetrizer matrix, once it is not less than the number $k$ of negative eigenvalues of the RH operator. The complete calculation requires $r$ columns of matrix $\mathbf{A}$, but if one stops at some other number of columns calculated, this is more or less successful approximation for the rank of this matrix.

The last step is application of the first, second, third, and subsequent Householder reflections and diagonalizations of corresponding matrices whose orders equal the number of reflection. Due to the variational character of these approximations, the results obtained, as usual, will converge to precise values from above.

The application of the method for noninteracting particles is the simplest and most transparent. In this situation, RH is proportional to the Hamiltonian, characteristic of any shell model picture. It is the Hamiltonian for one particle, moving in the external field, multiplied by the number of particles $N$, hence its eigenvalues are well-known one-particle energies multiplied 
by the same number. As it was mentioned, the matrix of the antisymmetrizer now is independent of any dynamics, and it splits down into a direct sum of projection matrices for different configurations. One is free to take spectral decompositions for every of these matrices separately. If particles are without any correlations, i.e., if the system can be described by one determinant, probabilities of all one-particle states in given configuration equal $1 / N$, hence the sum has a well-known form given in Eq. (26). In the case when one must ensure good quantum numbers for such a system, the probabilities of different states of RH will equal the squared coefficients of fractional parentage, obtained after spectral decomposition of the antisymmetrizer matrix.

Let us take for illustration of proposed ideas a simple model for ${ }^{1,3} F$ terms in $d^{4}$ configuration of four identical fermions with spin equal to $1 / 2$. The order $n$ of this matrix equals 8 , and the rank $r$ equals 3 . The matrix $\mathbf{F}$ of spectral decomposition is

$$
\mathbf{F}=\left(\begin{array}{ccc}
0 & -\sqrt{\frac{1}{15}} & -\sqrt{\frac{4}{15}} \\
\sqrt{\frac{3}{14}} & \sqrt{\frac{2}{105}} & -\sqrt{\frac{5}{42}} \\
0 & \sqrt{\frac{3}{8}} & 0 \\
\sqrt{\frac{5}{14}} & \sqrt{\frac{1}{56}} & \sqrt{\frac{1}{14}} \\
0 & \sqrt{\frac{4}{15}} & -\sqrt{\frac{1}{15}} \\
-\sqrt{\frac{3}{16}} & -\sqrt{\frac{1}{60}} & -\sqrt{\frac{5}{48}} \\
-\sqrt{\frac{3}{560}} & \sqrt{\frac{3}{28}} & -\sqrt{\frac{27}{112}} \\
-\sqrt{\frac{33}{140}} & \sqrt{\frac{11}{84}} & \sqrt{\frac{11}{84}}
\end{array}\right) .
$$

Let diagonal entries $\varepsilon_{i}$ of matrix of RH be

$$
-7 ;-4 ; 0 ; 0.1 ; 0.2 ; 0.3 ; 0.4 ; 0.5 \text {. }
$$

These eigenvalues are not far from the real situation. Existence of a few bound states of RH and all remaining states situated at positive energies, i. e., in the continuous spectrum, is characteristic of strong interaction. As mentioned, in our approach the continuous spectrum is discretized due to boundary conditions chosen.
The first state (column) of $\mathbf{F}$ matrix produces a very poor value for energy, equal to -0.645 , the second even worse, equal to -0.374 due to the smaller total weight of basic states corresponding to the first two negative eigenvalues of $\mathrm{RH}$. The result produced while applying the third state (column) of $\mathbf{F}$ is the best one. It equals -2.129 . This value is closest to the lowest eigenvalue of the total Hamiltonian matrix $\mathbf{F}^{+} \mathbf{H F}$, which equals -2.532 .

The result of the first Householder reflection, the first column of $\mathbf{F}^{\prime}=\mathbf{F R}_{1}$, gives the eigenvalue equal to -2.380 . After the second Householder reflection applied, the lowest eigenvalue of the second order matrix equals -2.532 , hence at a given precision it coincides with the exact eigenvalue of the total matrix of the Hamiltonian.

The abovementioned Hall-Post lower bound for this eigenvalue equals -7.000 . The diagonalization of the corresponding third order matrix in uncorrelated harmonic oscillator basis due to the core present in realistic nucleon-nucleon potential can give only positive values for the RH operator, hence a positive value for energy of the whole system. Obviously, our result is a real achievement for very complex interactions such as recent realistic nucleon-nucleon potentials.

Finally, let us draw some parallel between the developed approach and the well-known Hartree-Fock self-consistent field method. In both cases first of all the building blocks for the system wave function construction are necessary. The common feature in both approaches is construction of these blocks taking into account as many dynamic correlations as possible. In SCF these, due to the chosen scheme of simple antisymmetrization in given configuration, are the best possible one-particle functions. In our approach, when one can choose the refined way of antisymmetrization, these are the eigenfunctions of the reduced Hamiltonian operator. Both approaches at this stage have some attractive points. The SCF method applies determinants or CFP. The price for this simplicity is wellknown unavoidable appearance of the phenomenological central field, the conversion of the linear problem to nonlinear equation for one-particle wave functions, and the essential impossibility to take into account some dynamic correlations while applying this method. In our method, all dynamic correlations are taken into account in advance by using RH eigenfunctions, however now one can perform antisymmetrization only in infinite space. The next step in SCF is iterations for the self-consistent field and optimization of corresponding one-particle functions. In our approach, this cor- 
responds to the application of $k$ Householder reflections and diagonalizations of the Hamiltonian matrix of the growing order. This procedure is equivalent to the lowest configuration definition in the SCF method. Finally, if SCF approach in some cases does not work, superpositions of configurations are necessary. This essentially complicates the consideration. In our case in analogous situation, one needs to take more columns of the antisymmetrizer matrix, no more serious problems, no new complex operations. Our approach can be applied to the translationally invariant functions, while for the self-consistent field approach such a modification is impossible.

As a more realistic application let us comment on the modifications of traditional calculation in the harmonic oscillator basis for the three nucleon system [11]. The number of basic states (components) with the number of total oscillator quanta $E=44$ is 16744 . This equals the dimension of both - RH and antisymmetrizer - matrices. The rank of the antisymmetrizer matrix, equal to the dimension of the total Hamiltonian matrix, is 5553. The ground state energy of triton in this approximation for the realistic nucleon-nucleon potential Reid93 [12] equals $-7.59 \mathrm{MeV}$ (the exact value of ground state energy for this potential is -7.63). Among 5553 basic components only 131 have "actor" wave functions, corresponding to negative eigenvalues of RH in two nucleon channels $1^{+} 0\left({ }^{3} S_{1}-{ }^{3} D_{1}\right)$ and $0^{+} 1\left({ }^{1} S_{0}\right)$. Therefore, applying 130 Householder reflections for columns of matrix $\mathbf{F}$ before the total Hamiltonian matrix calculation one can transform it to a lower triangle form with only 131 columns having nonzero entries corresponding to negative eigenvalues of the RH operator. It is these entries that produce nonzero probabilities of corresponding states. As a result, only these 131 basic antisymmetric functions (transformed columns of matrix F) are necessary for construction of the total Hamiltonian matrix, ensuring the mentioned result for the triton ground state energy. Obviously, transformation of dimension of the Hamiltonian matrix from 5553 to 131 is a real achievement of the proposed method, giving opportunity to simplify the realistic nuclear calculations.

\section{Acknowledgement}

D. G. and R.-K. K. are grateful to the Lithuanian State Science and Studies Foundation in the frame of project No. T-12/06 for the partial support.

\section{References}

[1] A. Nogga, H. Kamada, and W. Glöckle, Modern nuclear force predictions for the alpha particle, Phys. Rev. Lett. 85, 944-947 (2000).

[2] R.B. Wiringa, S.C. Pieper, J. Carlson, and V.R. Pandharipande, Quantum Monte Carlo calculations of $A=8$ nuclei, Phys. Rev. C 62, 014001-1-23 (2000).

[3] P. Navratil, G.P. Kamuntavičius, and B.R. Barrett, Few-nucleon systems in a translationally invariant harmonic oscillator basis, Phys. Rev. C 61, 044001-1-16 (2000).

[4] G.P. Kamuntavičius, Simple functional-differential equations for the bound-state wave-function components, Few-Body Systems 1, 91-109 (1986).

[5] R.L. Hall and H.R. Post, Many-particle systems: IV. Short-range interactions, Proc. Phys. Soc. London 90, 381-396 (1967).

[6] G.P. Kamuntavičius, Metod reducirovannogo gamiltoniana $\mathrm{v}$ teorii svyazannykh sostoyaniy sistem tozhdestvennykh chastits, Fiz. Elem. Chastits At. Yadra 20, 261-292 (1989) [in Russian; in Eglish: Reduced-Hamiltonian method in the theory of bound states of systems of identical particles, Sov. J. Part. Nucl. 20, 109-122 (1989)].

[7] G. Racah, Theory of complex spectra III, Phys. Rev. 63, 367-382 (1943).

[8] U. Fano and G. Racah, Irreducible Tensorial Sets (Academic Press Inc., New York, 1959).

[9] A.J. Coleman, Structure of fermion density matrices, Rev. Mod. Phys. 35, 668-686 (1963).

[10] G. Strang, Linear Algebra (Academic Press, New York, 1988).

[11] G.P. Kamuntavičius, D. Germanas, R.-K. Kalinauskas, and R. Žemaičiūnienè, Lower bounds for energies of the bound states of an atomic nucleus, Lithuanian J. Phys. 43, 81-88 (2003).

[12] V.G.J. Stoks, R.A.M. Klomp, C.P.F. Terheggen, and J.J. de Swart, Construction of high-quality NN potential models, Phys. Rev. C 49, 2950-2962 (1994). 


\section{SUSIRIŠUSIU TAPATINGU FERMIONU KORELIACIJOS}

G.P. Kamuntavičius ${ }^{\text {a }}$, S. Mickevičius ${ }^{\text {a }}$, A. Mašalaitè ${ }^{a}$, D. Germanas ${ }^{b}$, R.K. Kalinauskas ${ }^{b}$, R. Žemaičiūnienė ${ }^{c}$

${ }^{\text {a }}$ Vytauto Didžiojo universitetas, Kaunas, Lietuva

${ }^{\mathrm{b}}$ Fizikos institutas, Vilnius, Lietuva

c Šiauliu universitetas, Šiauliai, Lietuva

\section{Santrauka}

Susirišusių tapatingų fermionų sistemoms, susidarančioms veikiant dvidalelinėms jègoms su bet kokio pavidalo potencialu, išplètotas universalus transliaciškai invariantinių antisimetrinių banginių funkcijų skaičiavimo metodas. Tarpdalelinès sąveikos sąly- gotos koreliacijos iskaitomos naudojant sistemos redukuotinio hamiltoniano tikrines funkcijas. Pasiūlytas tokių sistemų banginių funkciju antisimetrizavimo algoritmas suteikia galimybę antisimetrizacijos operaciją atlikti paprastu skaitmeniškai kontroliuojamu būdu. 